Chirurg 2013 $\cdot 84: 60$

DOI 10.1007/s00104-012-2436-1

Online publiziert: 14. Dezember 2012

c) Springer-Verlag Berlin Heidelberg 2012

T. Franzke $\cdot J$. Jähne

Klinik für Allgemein- und Viszeralchirurgie, Schwerpunkt für endokrine und onkologische Chirurgie,

Diakoniekrankenhaus Henriettenstiftung Hannover

\title{
Perioperative Sauerstoffgabe
}

\section{Vermeidung von Anastomoseninsuffizienzen nach Gastrektomie}

von 0,8 beatmet. Postoperativ wurde diese Trennung für $6 \mathrm{~h}$ aufrechterhalten (16 l $\mathrm{O}_{2} /$ min vs. Raumluft).

Schietroma M, Cecilia EM, Carlei F et al (2012) Prevention of anastomotic leakage after total gastrectomy with perioperative supplemental oxygen administration: a prospective randomized, double-blind, controlled, singlecenter trial. Ann Surg Oncol (Epub ahead of print)

Die Anastomoseninsuffizienz ist mit 7-15\% die gefürchtetste Komplikation nach Gastrektomie. Eine gute lokale Perfusion einhergehend mit einer guten Oxygenierung der ösophagojejunalen Anastomose ist die Grundvoraussetzung für die Heilung der Anastomose. Eine gute Gewebeoxygenierung und ein suffizienter Gasaustausch sind neben anderen Faktoren vom Sauerstoffpartialdruck im Blut abhängig. Ob die Rate an Anastomoseninsuffizienzen durch eine perioperative Sauerstoffgabe gesenkt werden kann, ist weiterhin unklar. Die vorliegende prospektive, randomisierte Studie untersucht diese Fragestellung an Patienten mit Gastrektomie.

\section{Material/Methodik}

In rund 3,5 Jahren wurden 171 Patienten (98 Männer, 73 Frauen) aufgrund einer malignen Erkrankung gastrektomiert und anschließend eine maschinelle Ösophagojejunostomie mittels Stapler (25 mm) durchgeführt. Nach der Narkoseeinleitung wurden 2 Gruppen randomisiert. Die eine Gruppe wurde mit einem $\mathrm{F}_{\mathrm{i}} \mathrm{O}_{2}$ von 0,3 und die andere mit einem $\mathrm{F}_{\mathrm{i}} \mathrm{O}_{2}$

\section{Ergebnisse}

Die Gesamtrate an Anastomoseninsuffizienzen lag bei 14,6\% (25/171). 17 (20\%) Patienten hatten eine Insuffizienz in der $0,3-\mathrm{F}_{\mathrm{i}} \mathrm{O}_{2}$-Gruppe und $8(9,3 \%)$ in der in der $0,8-\mathrm{F}_{\mathrm{i}} \mathrm{O}_{2}$-Gruppe $(\mathrm{p}<0,05)$. Das Risiko für eine Insuffizienz war in der $0,8-\mathrm{F}_{\mathrm{i}} \mathrm{O}_{2}$-Gruppe um $49 \%$ geringer als in der $0,3-\mathrm{F}_{\mathrm{i}} \mathrm{O}_{2}$-Gruppe. In der multivariaten Analyse zeigten sich ein hoher ASA (American Society of Anesthesiologists)Score, pulmonale Nebenerkrankungen sowie eine lange Operationszeit als weitere unabhängige Risikofaktoren. Die Gesamtmortalität lag bei 7\% (12/171), wobei 4 Patienten an den Folgen der Insuffizienz verstarben $(4 / 25=16 \%)-3$ dieser 4 Patienten gehörten zu der $0,3-\mathrm{F}_{\mathrm{i}} \mathrm{O}_{2}$-Gruppe. Weiterhin zeigte sich in $\operatorname{der} 0,8-\mathrm{F}_{\mathrm{i}} \mathrm{O}_{2}-$ Gruppe eine signifikant geringere Rate an Wundinfekten $(5,8 \%$ vs. $12,9 \%$; $\mathrm{p}<0,05)$.

\section{Diskussion}

Die vermehrte Gabe von Sauerstoff ist ein immer wiederkehrendes und weiterhin kontrovers diskutiertes Thema in der perioperativen Medizin. Viele Studien zeigten Vorteile (z. B. hinsichtlich einer geringeren Wunddehiszenzrate), andere Studien konnten dies nicht bestätigen und zeigten teilweise sogar Nachteile. Die vorliegende Arbeit liefert erstmals qualitativ gute Daten für eine vermehrte Sauerstoff- gabe während und $6 \mathrm{~h}$ nach einer Gastrektomie. Es konnte eine signifikant geringere Insuffizienz- und Wunddehiszenzrate in der $0,8-\mathrm{F}_{\mathrm{i}} \mathrm{O}_{2}$-Gruppe gezeigt werden.

Kritisch ist vor allem die mit $20 \%$ $(17 / 85)$ hohe Insuffizienzrate in der $0,3-\mathrm{F}_{\mathrm{i}} \mathrm{O}_{2}$-Gruppe zu hinterfragen, wobei die allgemein akzeptierte Rate (ohne Sauerstofftherapie) deutlich unter $10 \%$ liegen sollte.

Dennoch scheint die perioperativ vermehrte Gabe von Sauerstoff eine kostengünstige und nahezu nebenwirkungsfreie supportive Methode zur Verminderung postoperativer Komplikationen nach Gastrektomie zu sein und sollte zukünftig weiter untersucht werden. Eine allgemeine Empfehlung kann jedoch nicht ausgesprochen werden.

\section{Korrespondenzadresse}

\section{Dr. T. Franzke}

Klinik für Allgemein- und Viszeralchirurgie, Schwerpunkt für endokrine und onkologische Chirurgie, Diakoniekrankenhaus Henriettenstiftung Hannover, Marienstr. 72-90, 30171 Hannover Timm.Franzke@ddh-gruppe.de

Interessenkonflikt. Der korrespondierende Autor gibt für sich und seinen Koautor an, dass kein Interessenkonflikt besteht. 